\title{
RESEARCH
}

Open Access

\section{The characterization of extracellular vesicles-derived microRNAs in Thai malaria patients}

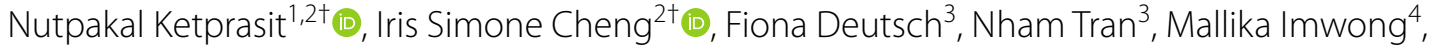 \\ Valery Combes ${ }^{2 *+}$ (D) and Duangdao Palasuwan ${ }^{5^{*}+}$ (1)
}

\begin{abstract}
Background: Extracellular vesicles (EVs) have been broadly studied in malaria for nearly a decade. These vesicles carry various functional biomolecules including RNA families such as microRNAs (miRNA). These EVs-derived microRNAs have numerous roles in host-parasite interactions and are considered promising biomarkers for disease severity. However, this field lacks clinical studies of malaria-infected samples. In this study, EV specific miRNAs were isolated from the plasma of patients from Thailand infected with Plasmodium vivax and Plasmodium falciparum. In addition, it is postulated that these miRNAs were differentially expressed in these groups of patients and had a role in disease onset through the regulation of specific target genes.
\end{abstract}

Methods: EVs were purified from the plasma of Thai P. vivax-infected patients $(n=19)$, P. falciparum-infected patients $(n=18)$ and uninfected individuals $(n=20)$. EV-derived miRNAs were then prepared and abundance of hsa-miR15b-5p, hsa-miR-16-5p, hsa-let-7a-5p and hsa-miR-150-5p was assessed in these samples. Quantitative polymerase chain reaction was performed, and relative expression of each miRNA was calculated using hsa-miR-451a as endogenous control. Then, the targets of up-regulated miRNAs and relevant pathways were predicted by using bioinformatics. Receiver Operating Characteristic with Area under the Curve (AUC) was then calculated to assess their diagnostic potential.

Results: The relative expression of hsa-miR-150-5p and hsa-miR-15b-5p was higher in P. vivax-infected patients compared to uninfected individuals, but hsa-let-7a-5p was up-regulated in both P. vivax-infected patients and P. falciparum-infected patients. Bioinformatic analysis revealed that these miRNAs might regulate genes involved in the malaria pathway including the adherens junction and the transforming growth factor- $\beta$ pathways. All up-regulated miRNAs could potentially be used as disease biomarkers as determined by AUC; however, the sensitivity and specificity require further investigation.

\footnotetext{
*Correspondence: valery.combes@uts.edu.au; nantadao@gmail.com

${ }^{\dagger}$ Nutpakal Ketprasit and Iris Simone Cheng are co-first authors

tValery Combes and Duangdao Palasuwan are co-senior authors

${ }^{2}$ Malaria and Microvesicles Research Group, School of Life Sciences,

Faculty of Sciences, University Technology of Sydney, Ultimo, Sydney,

NSW 2007, Australia

${ }^{5}$ Oxidation in Red Cell Disorders Research Unit, Department of Clinical

Microscopy, Faculty of Allied Health Sciences, Chulalongkorn University,

154 Rama 1 Road, Pathumwan, Bangkok 10330, Thailand

Full list of author information is available at the end of the article
}

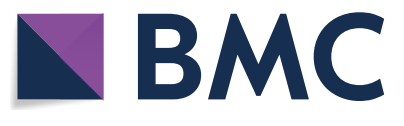

(c) The Author(s) 2020. This article is licensed under a Creative Commons Attribution 4.0 International License, which permits use, sharing, adaptation, distribution and reproduction in any medium or format, as long as you give appropriate credit to the original author(s) and the source, provide a link to the Creative Commons licence, and indicate if changes were made. The images or other third party material in this article are included in the article's Creative Commons licence, unless indicated otherwise in a credit line to the material. If material is not included in the article's Creative Commons licence and your intended use is not permitted by statutory regulation or exceeds the permitted use, you will need to obtain permission directly from the copyright holder. To view a copy of this licence, visit http://creativeco mmons.org/licenses/by/4.0/. The Creative Commons Public Domain Dedication waiver (http://creativecommons.org/publicdomain/ zero/1.0/) applies to the data made available in this article, unless otherwise stated in a credit line to the data. 
Conclusion: An upregulation of hsa-miR-150-5p and hsa-miR-15b-5p was observed in P. vivax-infected patients while hsa-let-7a-5p was up-regulated in both P. vivax-infected and $P$. falciparum-infected patients. These findings will require further validation in larger cohort groups of malaria patients to fully understand the contribution of these EVs miRNAs to malaria detection and biology.

Keywords: Malaria, Patients, Plasmodium falciparum, Plasmodium vivax, Extracellular vesicles, microRNAs

\section{Background}

According to the World Health Organization (WHO) World Malaria Report 2019, there were an estimated 405,000 deaths, and 228 million cases were reported globally [1]. Plasmodium vivax has the highest prevalence and is known to cause relapse infection [2], whereas $P$. falciparum is the most virulent species causing severe syndromes. Malaria appears to be more devastating worldwide as the emergence of artemisinin-resistant malaria parasites [3, 4]. To overcome the disease, novel drug development is essential. On the other hand, indepth understanding of the parasite biology and mechanisms underlying the disease is also urgently needed.

Extracellular vesicles (EVs), which are small membrane-bound vesicles, have been explored in malaria for the last decade. Classification of EVs is based on their cellular origin, size, and biological functions [5]. Two major types of EVs have been studied broadly including microvesicles (previously named microparticles) and exosomes. Another type of EVs are the apoptotic bodies which are released during the apoptotic process [6]. Microvesicles are vesicles that bleb from the cell membrane whereas exosomes are released from multivesicular bodies (MVBs) through an exocytotic process in the endolysosomal pathway [7].

Typically, EVs can be detected in very low numbers in healthy individuals. However, upon activation which is triggered by various pathological conditions [8-11], the number of EVs present in biological fluids increases. Various biomolecules were identified in EVs such as proteins, lipids, and nucleic acids [12]. Such biomolecules entrapped in EVs play many important roles in intercellular communication in numerous diseases, including malaria $[13,14]$.

EVs numbers have been shown to increase during malaria infection both in patients and in experimental malaria models $[10,11,15-18]$. They are associated with either protective [19] or enhanced pathogenicity of malaria infection $[11,16-18,20,21]$. The most common feature demonstrated in malaria is that EVs can act as immunomodulators. EVs from infected-erythrocytes can stimulate innate immune cells including macrophages [22], natural killer cells [23], monocytes and neutrophils [24]. Interestingly, EVs participate in cell-cell communication between parasites and parasites or host cells. Exosome-like vesicles have played a role in gametocytogenesis, which is crucial for malaria transmission, and these small membrane-bound vesicles could transfer drug-resistance markers to drug-sensitive parasites [25]. Additionally, EVs which cargo P. falciparum lactate dehydrogenase (PfLDH) could control parasite density in vitro [26]. One study also showed that EVs enriched with the parasite's genomic DNA, released from infected red blood cells could be internalized by monocytes and elicited an innate immune response [27].

As mentioned above, EVs carry many kinds of biomolecules including microRNAs (miRNAs). EVs were shown to protect the miRNAs from RNases-mediated degradation and EVs-entrapped miRNAs were also demonstrated to have regulatory functions [28-30]. MiRNAs are members of the non-coding RNAs family which were first discovered in Caenorhabditis elegans [31]. Malaria parasites do not express specific miRNAs [32, 33], as Dicer or Argonaut encoding genes are not found in these parasites [34-36]. However, several studies showed that miRNAs could translocate to P. falciparum [37, 38]. A pioneer investigation by Lamonte et al. showed interaction between human miRNAs and P. falciparum, where the expression of hsa-miR-451a and hsa-let-7i were increased in sickle cells. These miRNAs could inhibit parasitic protein translation resulting in low proliferation of parasites [38]. In other studies, expression of miRNAs in heart and brain tissues from mice with cerebral malaria were shown to be dysregulated when compared to noncerebral malaria $[39,40]$.

MiRNAs, human Argonaute protein and RISC complex could be detected in the parasites [37] as well as in EVs released by infected erythrocytes [41, 42]. EVs carried Argonaut-miRNAs complex affecting recipient cells that have been demonstrated to alter vascular function [41] or the parasite's var gene expression [43]. These studies strengthen the roles of EVs-derived miRNAs in malaria pathogenesis. There are only three studies that have analysed miRNAs in malaria-infected patients. In human plasma samples, the expression of hsa-miR-451a and hsa-miR-16 in $P$. vivax-infected patients were lower than those in $P$. falciparuminfected patients. Moreover, hsa-miR-451a and hsamiR-16 had a negative relationship with parasitaemia [44]. In a post-mortem study, the expression of various 
miRNAs were found to be differentially expressed between malaria and non-malaria deaths [45]. Furthermore, hsa-miR-146a was newly predicted to play a role in innate immunity in pregnancy malaria [46]. Taken together, these studies highlight the potential roles of miRNAs in malaria infection.

Despite the fact that the presence of EVs-derived miRNAs has been demonstrated both in vitro and in experimental cerebral malaria [47], according to the literatures available, no study has analysed EVs-derived miRNAs in human plasma. Thus, this present study examined the relative expression of selected miRNAs isolated from human plasma EVs. In the present study, the relative expression among three biological groups was compared, i.e., $P$. vivax-infected or $P$. falciparum-infected patients and uninfected individuals. The whole population of circulating EVs, namely microvesicles together with exosomes were analysed to gain a better understanding of the whole EV-bound miRNA population and to not restrict this analysis to one sub-population. By studying the EV compartment as a whole the chances of finding differences in miRNA expression between the groups would be increased. Five miRNA were selected based on previous in vitro, animal model, or clinical studies that suggested these miRNAs had a potential involvement in malaria, namely: hsa-miR-451a, hsa-miR-150-5p, hsa-miR-15b-5p, hsa-let-7a, and hsa-miR-16-5p [37-39, $41-44,48]$. This study provides novel insight about circulating EVs-derived miRNAs in human malaria.

\section{Methods}

\section{Human samples collection and preparation}

Malaria patients with $P$. falciparum or $P$. vivax were confirmed by microscopic examination by medical laboratory scientists at Buntharik district hospital in Ubon Rachathani. (Ethics approval by Ethics committee of the Faculty of Tropical Medicine, Mahidol University, MUTM 2012-046-05). Parasite species were identified and parasitaemia of each sample was calculated by counting the number of malaria-infected erythrocytes over the number of normal erythrocytes per thousand cells. Uninfected donors were recruited, and venipuncture was performed for all donors using tri-potassium ethylenediaminetetraacetic acid (K3EDTA) as an anticoagulant. K3EDTA blood samples were then centrifuged at $1500 \times g$ for $15 \mathrm{~min}$.

Then, platelet-free plasma samples were prepared by centrifugation at $13,000 \times g$ for $2 \mathrm{~min}$ at room temperature (RT) [17]. Supernatants were collected and stored at $-80{ }^{\circ} \mathrm{C}$ for further isolation of EVs. Samples were then shipped to the University of Technology Sydney where all experiments were performed.

\section{Extracellular vesicles isolation from human plasma}

$500 \mu \mathrm{L}$ of supernatants were mixed with $200 \mu \mathrm{L}$ of sodium citrate and $300 \mu \mathrm{L}$ of phosphate buffered saline (PBS) and then centrifuged at $150,000 \times g$ for $3 \mathrm{~h}$ at $15{ }^{\circ} \mathrm{C}$. Supernatants were discarded and pellets were resuspended in $100 \mu \mathrm{L}$ sodium citrate and $900 \mu \mathrm{L}$ PBS and subjected to centrifugation at $150,000 \times g$ for $3 \mathrm{~h}$ at $15{ }^{\circ} \mathrm{C}$.

\section{Total RNA extraction}

Pellets obtained after centrifugation were homogenized with $1 \mathrm{~mL}$ of RNAzol (Molecular Research Center, Inc) and $400 \mu \mathrm{L}$ of UltraPure ${ }^{\mathrm{TM}}$ DNase/RNase-Free distilled water (Invitrogen ${ }^{\mathrm{TM}}$ ). After a five-minute incubation at RT, they were centrifuged for $15 \mathrm{~min}$ at $12,000 \times g$ at $4{ }^{\circ} \mathrm{C}$. Supernatants were transferred to new tubes with $800 \mu \mathrm{L}$ isopropanol and $5 \mu \mathrm{L}$ glycogen $(5 \mathrm{mg} / \mathrm{mL})$ then gently mixed. They were then placed at $-30{ }^{\circ} \mathrm{C}$ overnight and spun for $10 \mathrm{~min}$ at $12,000 \times g$ at $4{ }^{\circ} \mathrm{C}$ the next day. Pellets were kept and washed with cold $75 \%$ ethanol to remove excess isopropanol, spun at $9000 \times g$ for $3 \mathrm{~min}$ at $4{ }^{\circ} \mathrm{C}$, the supernatant was discarded, and these steps were repeated twice. Later, excess ethanol was removed by spinning at $12,000 \times g$ for $30 \mathrm{~s}$. RNA pellets were resolubilized with $10 \mu \mathrm{L}$ of UltraPure ${ }^{\mathrm{TM}}$ DNase/ RNase-Free distilled water and heated for $5 \mathrm{~min}$ at $55^{\circ} \mathrm{C}$. Lastly, they were vortexed and briefly spun down.

\section{Total RNA concentration measurement and cleaning}

RNA concentration was measured using the NanoDrop One Microvolume UV-Vis Spectrophotometer (Thermo Scientific $^{\mathrm{TM}}$ ). If the quality of total RNA in the samples was not optimal, i.e., the Nanodrop flagged the result as phenol contamination or Absorbance 260/230<1.7, a sodium acetate $(\mathrm{NaOAc})$, a cleaning step was performed. Briefly, $5 \mu \mathrm{L}$ of glycogen $(5 \mathrm{mg} / \mathrm{mL})$ and $2.5 \mu \mathrm{L}$ of sodium acetate (3 M pH5.5) were added to samples and mixed comprehensively. The mixture was supplemented with $110 \mu \mathrm{L}$ of absolute ethanol and incubated overnight in $-30{ }^{\circ} \mathrm{C}$. Later, washing steps were performed as mentioned in the RNA extraction with RNAzol above.

\section{Primers for miRNAs detection}

All primers in this study was purchased from Thermo Fisher Scientific.

Five human miRNAs were used to perform quantitative polymerase chain reaction as described in Table 1.

\section{Reverse transcriptase quantitative polymerase chain reaction (RT-qPCR)}

As the NanoDrop was not sensitive enough to measure the RNA concentration, fixed volumes of RNA for 
Table 1 Primers used for quantitative PCR

\begin{tabular}{lll}
\hline miRNAs & $\begin{array}{l}\text { NCBI accession } \\
\text { number }\end{array}$ & Mature miRNAs sequences \\
\hline hsa-miR-451a & Ml0001729 & AAACCGUUACCAUUACUGAGUU \\
hsa-miR-15b-5p & M10000438 & UAGCAGCACAUCAUGGUUUACA \\
hsa-miR-16-5p & M10000070 & UAGCAGCACGUAAAUAUUGGCG \\
hsa-let-7a-5p & Ml0000060 & UGAGGUAGUAGGUUGUAUAGUU \\
hsa-miR-150-5p & Ml0000479 & UCUCCCAACCCUUGUACCAGUG \\
\hline
\end{tabular}

cDNA synthesis were used. To analyse miRNAs expression, $0.5 \mu \mathrm{L}$ of total RNA was used for cDNA synthesis. RT-qPCR was performed as per manufacturer protocol using TaqMan ${ }^{\circledR}$ fast advanced master mix and TaqMan ${ }^{\circledR}$ advanced miRNA assay. Quantitative PCR (qPCR) was run on the QuantStudio 6 flex system (Applied Biosystem) in triplicate together with distilled water as negative control.

\section{Relative expression analysis}

Only samples that had not been already freeze-thawed were included in the study to avoid RNA degradation. Comparative threshold cycle or quantification cycle $\mathrm{C}_{\mathrm{q}}$ (as described in [49]) method was used to analyse the qPCR data. Each sample was run in triplicates. Then, the average $C_{q}$ from the three $C_{q}$ values of those samples was calculated. To select one miRNA as an endogenous control, mean, standard deviation and variance for each miRNA analysed were calculated. $\Delta C_{q}$ was then calculated using the following equation $\Delta \mathrm{C}_{\mathrm{q}}=\mathrm{C}_{\mathrm{q}}$ of miRNA $-\mathrm{C}_{\mathrm{q}}$ of endogenous control and relative expression was calculated by $2^{(-\Delta \Delta \mathrm{Cq})}[50]$.

\section{Target prediction and pathway involvement of dysregulated miRNAs}

To predict possible targets of up-regulated miRNAs, the miRNAs of interest was submitted to Targetscan Release 7.2 and the predicted target genes were retrieved [51]. Then, the targets that overlapped genes involved in malaria pathway were obtained from the Kyoto Encyclopedia of Genes and Genomes (KEGG) [52]. In order to identify the pathways for individual and combined analysis of miRNAs, DIANA-mirPath v3.0 was used, with a 5\% false discovery rate (FDR) [53]. Fisher's exact test (Hypergeometric distribution) was applied for enrichment analysis. The potential pathways were considered based on their $p$ value $(\mathrm{p}<0.05)$.

\section{Evaluation of potential EVs-derived miRNA as biomarker} Mean of Delta Cq for each miRNA was used in this analysis following the previous study [54]. The receiver operating characteristic (ROC) was calculated to propose the potential use of miRNAs as diagnostic tools. Area under the curve (AUC) was analysed to show the accuracy of the test with p-value of each analysis.

\section{Statistical analysis}

For the samples, demographic, mean age of each group was presented by average and standard deviation. All data was tested for their normality by using Kolmogorov-Smirnov or Shapiro-Wilk tests and a non-parametric statistical analysis method was chosen. Comparison of parasitaemia percentage was achieved by the MannWhitney U Test. Kruskal-Wallis test was used for comparison of relative expression of miRNAs in each biological group followed by post hoc analysis using Dunn's test. Statistical significance for all tests was considered significant for $\alpha=0.05$. All statistical tests were analysed by Prism 8 . All figures shown in this article were created by Prism 8 software for Mac, GraphPad Software, La Jolla California USA, https://www.graphpad.com.

\section{Results}

\section{Patient's characteristics}

57 plasma samples were included in this study divided into three groups; $P$. vivax-infected patients $(\mathrm{n}=19)$, P. falciparum-infected patients $(\mathrm{n}=18)$, and uninfected community individuals $(\mathrm{n}=20)$ as per Table 2 . The average age of malaria patients (Mean $\pm \mathrm{SD}$ ) was

Table 2 Sample characteristic

\begin{tabular}{llll}
\hline Characteristics & PV-infected patients & PF-infected patients & Uninfected \\
\hline $\begin{array}{l}\text { Total number of patients } \\
\text { Gender }\end{array}$ & 19 & 18 & 20 \\
Male & 16 & 17 & 11 \\
Female & 3 & 1 & 9 \\
Age (Mean \pm SD)* & $31.6 \pm 9.59$ & $33.4 \pm 11.91$ & $21.8 \pm 0.89$ \\
$\%$ Parasitaemia (Mean \pm SD) ${ }^{* *}$ & $0.35 \pm 0.28$ & $1.29 \pm 1.48$ & \\
\hline
\end{tabular}

* Mean and SD of age $P$. vivax-infected patients was calculated from 10 patients, Mean and SD of age of PF-infected patients was calculated from 15 patients

** \%parasitaemia of $P$. vivax-infected patients calculated from 16 patients. \%parasitaemia of PF-infected patients calculated from 14 patients 
$32.68 \pm 11.08$ years, $P$. vivax-infected patients was $31.6 \pm 9.59$, and $P$. falciparum-infected patients was $33.4 \pm 11.91$. The average age of uninfected individuals $(21.8 \pm 0.89$ years $)$ was significantly lower than both $P$. vivax-infected patients or P. falciparum-infected patients $(\mathrm{p}=0.018, \mathrm{p}=0.001$, respectively). Percentage of parasitaemia (\% Parasitaemia) of P. VIVAX-infected patients was $0.35 \pm 0.28$ while $P$. falciparum-infected patients was $1.29 \pm 1.48$. Overall parasitaemia was higher in $P$. falciparum-infected patients than $P$. vivax-infected patients $(\mathrm{p}=0.0115)$ as shown in Fig. 1a.

\section{Relative expression of miRNAs in three biological groups}

Total RNA was extracted from EVs pellets and analysed hsa-miR-451a, hsa-miR-15b-5p, hsa-miR-16-5p, hsalet-7a-5p and hsa-miR-150-5p among $P$. vivax-infected patients, $P$. falciparum-infected patients and uninfected individuals. The average $\mathrm{C}_{\mathrm{q}}$ was calculated from triplicate run (Fig. 1b). Hsa-miR-451a had the lowest $C_{q}$ with the minimum standard deviation compared to the other miRNAs, at $26.36 \pm 2.33$ (Mean \pm SD). A Kruskal-Wallis test was undertaken to compare the $\mathrm{Cq}$ values in each biological group and showed that the values were not statistically different. These results indicated that this miRNA was the most stable among three biological groups and could be used as internal control. Moreover, previous studies demonstrated that hsa-miR-451a was highly expressed in both uninfected and parasitized red blood cells [32] and its expression was independent on the intra-erythrocytic development of the malaria parasite [33].

In the present study, EVs were isolated from Thai patients presenting with uncomplicated malaria and demonstrated that miRNAs can be detected in these EVs. Descriptive statistics of $\mathrm{Cq}$ values are presented in Table 3. The relative expression of each miRNA was calculated using hsa-miR-451a as endogenous control using the $2^{(-\Delta \Delta \mathrm{Cq})}$ method [50]. The relative expressions of hsa-miR-15b-5p, hsa-miR-16-5p, hsa-let-7a-5p and hsa-let-7a-5p were compared between malariainfected (regardless of the parasite species) and uninfected individuals (Fig. 2). Hsa-miR-150-5p ( $\mathrm{p}=0.0054)$,
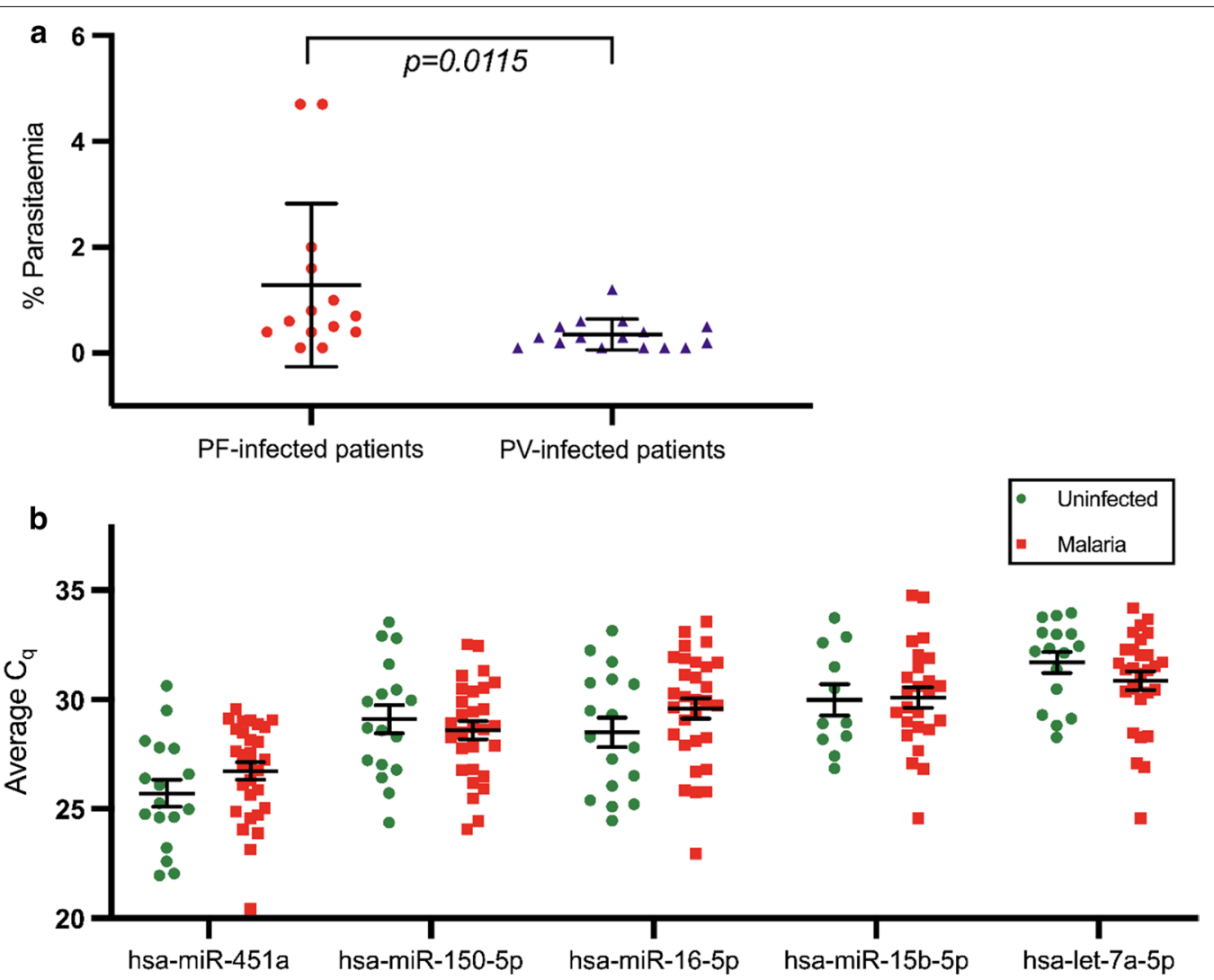

Fig. 1 a Malaria infected patients with P. vivax or P. falciparum calculated as a percentage of parasitaemia. The average of parasitaemia was tested by Mann-Whitney U Test, which showed that the percentage of parasitaemia of those infected with $P$. falciparum is significantly higher than those with P. vivax ( $p=0.0115)$. b miRNAs isolated from plasma-derived EVs were analysed by RT-qPCR (TaqMan ${ }^{\mathrm{TM}}$ Advanced miRNA Assays) with specific primers for hsa-miR-451a, hsa-miR-15b-5p, hsa-miR-16-5p, hsa-let-7a-5p and hsa-miR-150-5p. The plots show the average Cq values for each miRNA, and these values are compared between malaria infected patients and uninfected individuals. (Presented as standard error of mean, SEM) 
Table 3 Descriptive statistics of quantification cycle $\left(C_{q}\right)$ values

\begin{tabular}{lllll}
\hline miRNAs & Min Cq & Max Cq & Mean \pm SD & $\begin{array}{l}\text { Coefficient } \\
\text { of variation } \\
\text { (\%) }\end{array}$ \\
\hline hsa-miR-451a & 20.43 & 30.63 & $26.36 \pm 2.33$ & 8.84 \\
hsa-miR-15b-5p & 24.57 & 34.77 & $30.06 \pm 2.34$ & 7.77 \\
hsa-miR-16-5p & 22.96 & 33.57 & $29.20 \pm 2.65$ & 9.09 \\
hsa-let-7a-5p & 24.57 & 34.19 & $31.17 \pm 2.15$ & 6.91 \\
hsa-miR-150-5p & 24.07 & 33.54 & $28.78 \pm 2.37$ & 8.24 \\
\hline
\end{tabular}

hsa-miR-15b-5p $\quad(p=0.0053)$ and hsa-let-7a-5p $(p=0.0002)$ were significantly up-regulated in EVs from malaria-infected patients comparing to the uninfected group. However, the relative expression of hsa-miR-16-5p between infected and non-infected individuals was not different.

Next, the changes in the abundance of these miRNAs were examined among the three biological groups (Fig. 3). An up-regulation of hsa-miR-150-5p ( $=0.0119)$ and hsa-miR-15b-5p $(\mathrm{p}=0.0052)$ was observed in EVs from $P$. vivax-infected patients. Remarkably, hsa-let$7 \mathrm{a}-5 \mathrm{p}$ expression was higher in both $P$. vivax-infected patients and $P$. falciparum-infected patients $(\mathrm{p}=0.0025$, $\mathrm{p}=0.0145$, respectively). When those parameters were compared between $P$. vivax-infected patients and $P$. falciparum-infected patients, there was no difference. The descriptive analysis of each miRNA in each group is presented in Table 4.

\section{Target prediction and KEGG Pathway analysis of hsa-miR150-5p and hsa-miR-15b-5p}

In order to study the possible roles of up-regulated miRNAs in malaria patients, the prediction was made using Targetscan. These targets were later overlapped with genes involved in the biological pathways that are relevant to malaria. There were 15, 5, and 6 targets of hsa-miR-150-5p, hsa-miR-15b-5p, and hsa-let-7a-5p, respectively as shown in Additional file 1: Table S1. Some targets were regulated by more than one miRNA of interest. Hepatocyte growth factor (HGF) encoding gene was regulated by all up-regulated miRNAs. Toll-like receptor 4 (TLR4), interleukin 10, and thrombospondin- 1 were found regulated by hsa-miR-150-5p and hsa-let-7a-5p.

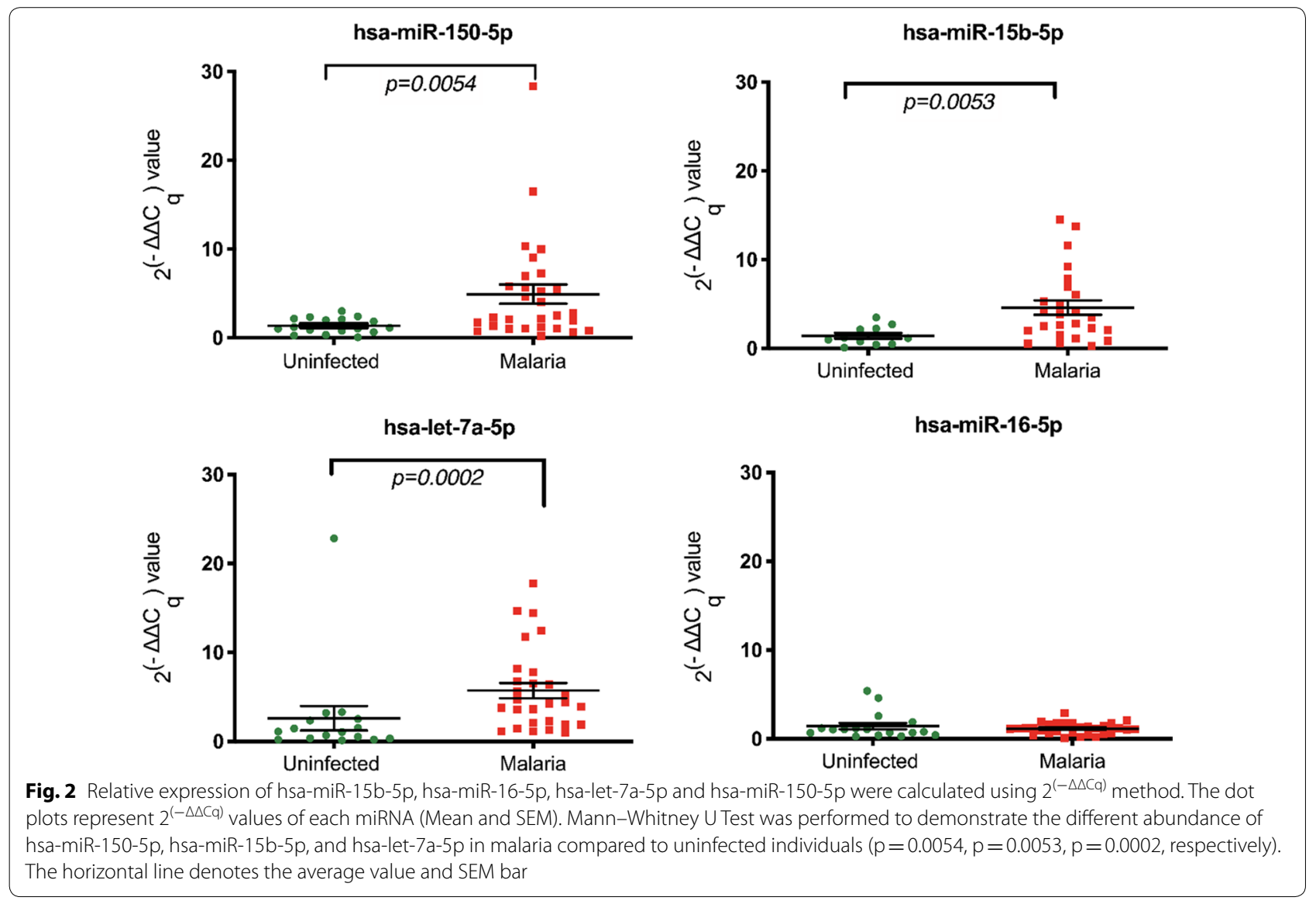



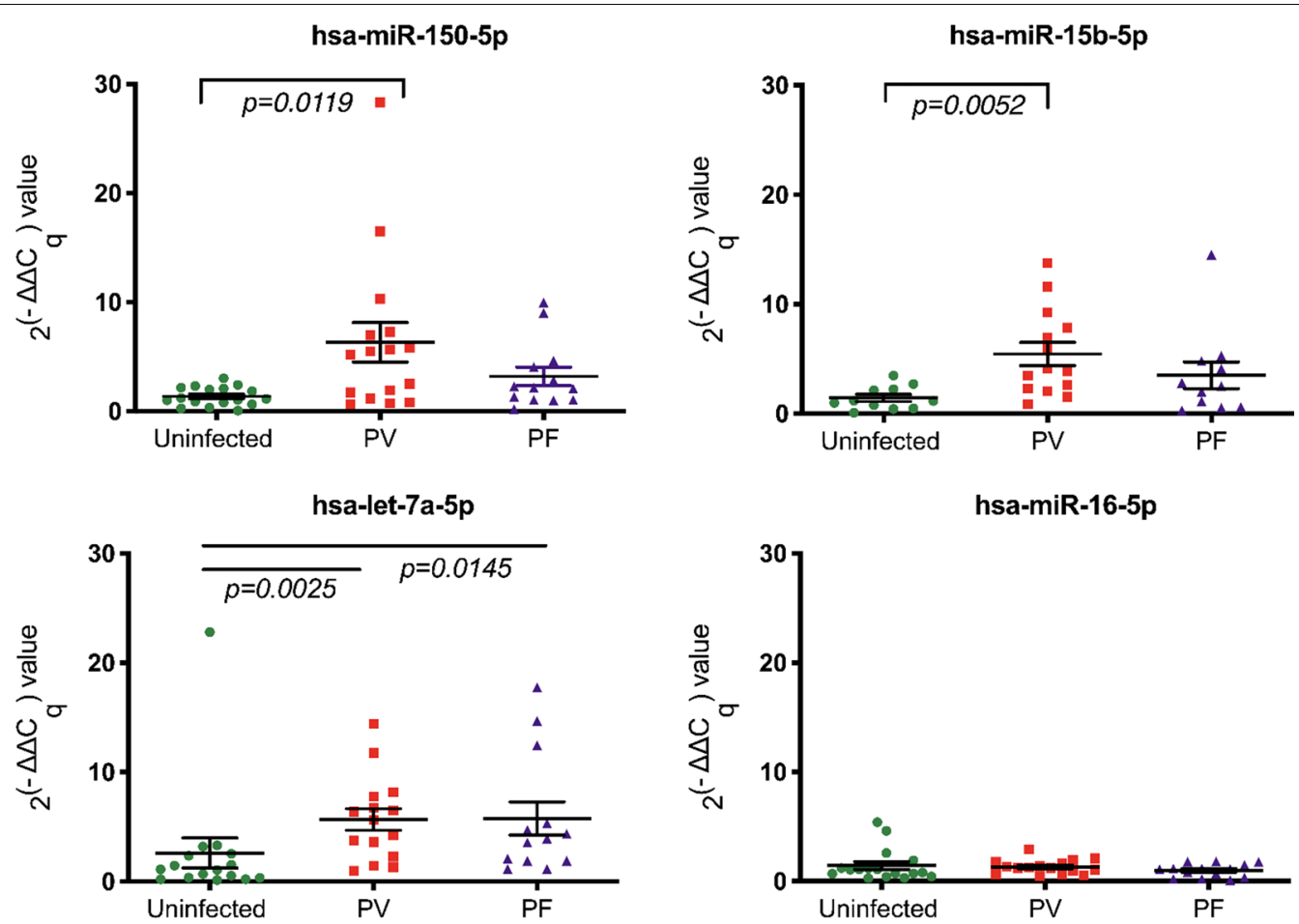

Fig. 3 This dot plot demonstrates the miRNAs expression values in 3 biological groups (Uninfected, $P$. vivax-infected patients or $P$. vivax, and $P$. falciparum-infected patients or P. falciparum). The Kruskal-Wallis was tested and followed by post hoc analysis using Dunn's test. hsa-miR-150-5p, hsa-miR-15b-5p were significantly up-regulated in $P$. vivax $(p=0.0119, p=0.0052$, respectively). Relative expression of hsa-let-7a-5p was higher in both $P$. vivax and $P$. falciparum ( $p=0.0025, p=0.0145$, respectively). The horizontal line denotes the average value and SEM bar

Table 4 Relative expression analysis of miRNAs

\begin{tabular}{|c|c|c|}
\hline & $\Delta C_{q}($ Mean $\pm S D)$ & $\begin{array}{l}2^{(-\Delta \Delta C)}{ }^{q} \text { Mean } \\
(\text { Mean } \pm S D)\end{array}$ \\
\hline \multicolumn{3}{|l|}{ hsa-let-7a-5p } \\
\hline Uninfected & $6.23 \pm 1.91$ & $1.28 \pm 1.10$ \\
\hline PV & $4.09 \pm 1.17$ & $5.68 \pm 3.85$ \\
\hline PF & $4.26 \pm 1.32$ & $5.76 \pm 5.52$ \\
\hline \multicolumn{3}{|c|}{ hsa-miR-15b-5p } \\
\hline Uninfected & $5.34 \pm 1.49$ & $1.43 \pm 1.07$ \\
\hline PV & $3.29 \pm 1.17$ & $5.45 \pm 3.96$ \\
\hline PF & $4.33 \pm 1.68$ & $3.51 \pm 4.04$ \\
\hline \multicolumn{3}{|l|}{ hsa-miR-16-5p } \\
\hline Uninfected & $2.79 \pm 1.22$ & $1.44 \pm 1.47$ \\
\hline PV & $2.56 \pm 0.72$ & $1.31 \pm 0.64$ \\
\hline PF & $3.21 \pm 1.32$ & $1.00 \pm 0.60$ \\
\hline \multicolumn{3}{|c|}{ hsa-miR-150-5p } \\
\hline Uninfected & $3.39 \pm 1.44$ & $1.37 \pm 0.86$ \\
\hline PV & $1.54 \pm 1.64$ & $6.32 \pm 7.23$ \\
\hline PF & $2.33 \pm 1.49$ & $3.20 \pm 3.07$ \\
\hline
\end{tabular}

Next, to get insight into these up-regulated miRNAs, a KEGG pathway analysis was performed using DIANAmirPath v3.0 that searches against experimentally validated miRNAs targets on Tarbase v7.0. The pathway analysis revealed 10,22 , and 32 pathways that were statistically overrepresented by targeted genes of hsa-miR150-5p, hsa-miR-15b-5p, and hsa-let-7a-5p, respectively. When the combined analysis of up-regulated miRNAs was determined, there were 44 enriched pathways. Of those enrichment pathways, the overlapped pathways included adherens junction (5th in the list, $\mathrm{p}=2.52 \mathrm{E}^{-10}$ ) as well as TGF-beta signaling pathway (11th in the list, $\left.\mathrm{p}=1.64 \mathrm{E}^{-7}\right)$. Other important pathways that might engage in malaria were extracellular matrix (ECM)receptor interaction (14th in the list, $\mathrm{p}=0.0005$ ), FoxO signaling pathway (29th in the list, $\mathrm{p}=0.0332$ ) and HIF-1 signaling pathway (30th in the list, $\mathrm{p}=0.0353$ ). In Fig. 4 , the top 10 enriched pathways analysis were presented according to their ranks based on p-value.

\section{Evaluation of the diagnostic potential of extracellular vesicles-derived miRNAs}

In addition, this study aimed to investigate whether the EVs-bound miRNAs could be used as putative diagnostic 
(See figure on next page.)

Fig. 4 Pathway enrichment analysis of up-regulated miRNAs were carried out by DIANA-mirPath v3.0 which searching against experimentally validated miRNAs targets on Tarbase v7.0. The combinatorial effect of up-regulated miRNAs was determined. Of those enriched pathways, the malaria-relevant pathways was found including adherens junction and TGF-beta signaling pathway. The bar chart presents with top 10 enriched pathway. The horizontal lines present - $\log 10$ p-values

markers. The ROC was used, and the AUC was calculated for each miRNA in both $P$. falciparum-infected patients and $P$. vivax-infected patients. Three miRNAs showed a statistical significance in the $P$. vivax-infected patients. hsa-miR-150-5p AUC was $0.7794(\mathrm{p}=0.0062)$, hsa-miR15b-5p AUC was $0.8766(\mathrm{p}=0.0015)$ and hsa-let-7a was $0.8375(\mathrm{p}=0.0014)$. In the $P$. falciparum-infected patients, only hsa-let-7a-5p was statistically significant with AUC $0.8221(\mathrm{p}=0.0033)$. Area under the ROC was shown with 95\% confidence interval value (Fig. 5).

\section{Discussion}

The present study, demonstrated that miRNAs can be detected in EVs were isolated from the blood of both Thai patients presenting with uncomplicated malaria and healthy donors. The miRNAs selected included hsa-miR451a, hsa-miR-15b-5p, hsa-miR-16-5p, hsa-let-7a-5p and hsa-miR-150-5p that were previously analysed in the context of malaria [37-39, 41-44, 48]. As mentioned earlier, hsa-miR-451a was selected as endogenous control as its $\mathrm{Cq}$ values were the most stable among three groups. In contrast, Chamnanchanunt et al. found that hsa-miR-451a was down-regulated in P. vivax-infected patient plasma [44]. Other studies demonstrated that EVs cargo hsa-miR-451a could be internalized to target the parasites and diminish the parasite burden [38, 43]. Furthermore, in an in vitro study, red blood cell derived EVs containing hsa-miR-451a and human argonaute 2 (Ago2) were shown to be internalized by endothelial cells. This miR-Ago2 could down-regulate the expression of CAV-1 and ATF2 resulting in endothelial cells alteration which is a plausible factor contributing vascular dysfunction in cerebral malaria [42]. As various studies showed that EVs that cargo hsa-miR-451a could be taken up by various cells in the context of malaria, it would, therefore, be interesting to enumerate the number of $\mathrm{EVs}$ and compare it with the change in abundance of hsa-miR-451a.

The hsa-miR-15b-5p and hsa-miR-150-5p were upregulated in the plasma-derived EVs from $P$. vivaxinfected patients but not in the P. falciparum-infected patients. An analysis of miRNAs from whole blood of adult imported falciparum malaria showed a down regulation of hsa-miR-150-5p [48]. Kaur et al. have identified a potential biomarker for differential diagnosis between uncomplicated and complicated $P$. vivax malaria infection, hsa-miR-7977. In addition, an increase in this
miRNA was predicted to be involved in malaria pathogenesis through the transforming growth factor beta (TGF- $\beta$ ) signaling pathway [48]. Recently, a study in nonhuman primate (Aotus lemurinus lemurinus) confirmed that bone marrow is an important reservoir for gametocytogenesis and proliferation of $P$. vivax [55]. A study in bone marrow aspirate of human diagnosed with $P$. vivax showed an aberrant expression of miRNAs in CD71 positive erythroid cells during infection, hsa-miR-150 and hsa-miR-16 were down-regulated while hsa-miR-144 was increased. However, hsa-miR-451a, the most abundant erythroid miRNA, remained stable [56]. In experimental cerebral malaria (ECM), an investigation in brain tissue found a higher expression of miR-150 while microvesicles from ECM mice showed non statistically different change of miR-150 expression [39, 47]. Nonetheless, this present study only investigated the plasma from the patients before drug administration and the clinical manifestation after treatment were not followed. Taken together, these data suggest that hsa-miR-150-5p might be an essential miRNA involved in malaria infection.

The relative expression of hsa-miR-15b-5p was higher in EVs from $P$. vivax-infected patients. To date, there is no evidence about the altered expression of this miRNA which might link to $P$. vivax malaria. However, this miRNA is present in P. falciparum in vitro and its abundance was decreased following infection. Also, it was potentially predicted to form a chimeric fusion with ringinfected erythrocyte surface antigen (RESA) [38]. Even though the relative expression of this miRNA was not statistically significant in $P$. falciparum-infected patients, it was noticed in this study that it was slightly higher when this miRNA was compared with the uninfected group.

Next, the relative expression of hsa-let-7a, which was found in infected erythrocytes [33], was determined. The relative abundance of hsa-let-7a was significantly increased in both $P$. vivax-infected patients and $P$. falciparum-infected patients compared to uninfected controls. these results are in accordance with previous studies showing that this miRNA could be detected in EVs and might be derived from parasitized red blood cells $[41,42]$. It is noteworthy that the overall expression of this miRNA is higher in the malaria patients (regardless of the parasite) than in uninfected donors. Several studies demonstrated that hsa-let-7a plays a role in hostparasite interaction $[37,41]$. This miRNA in complex 

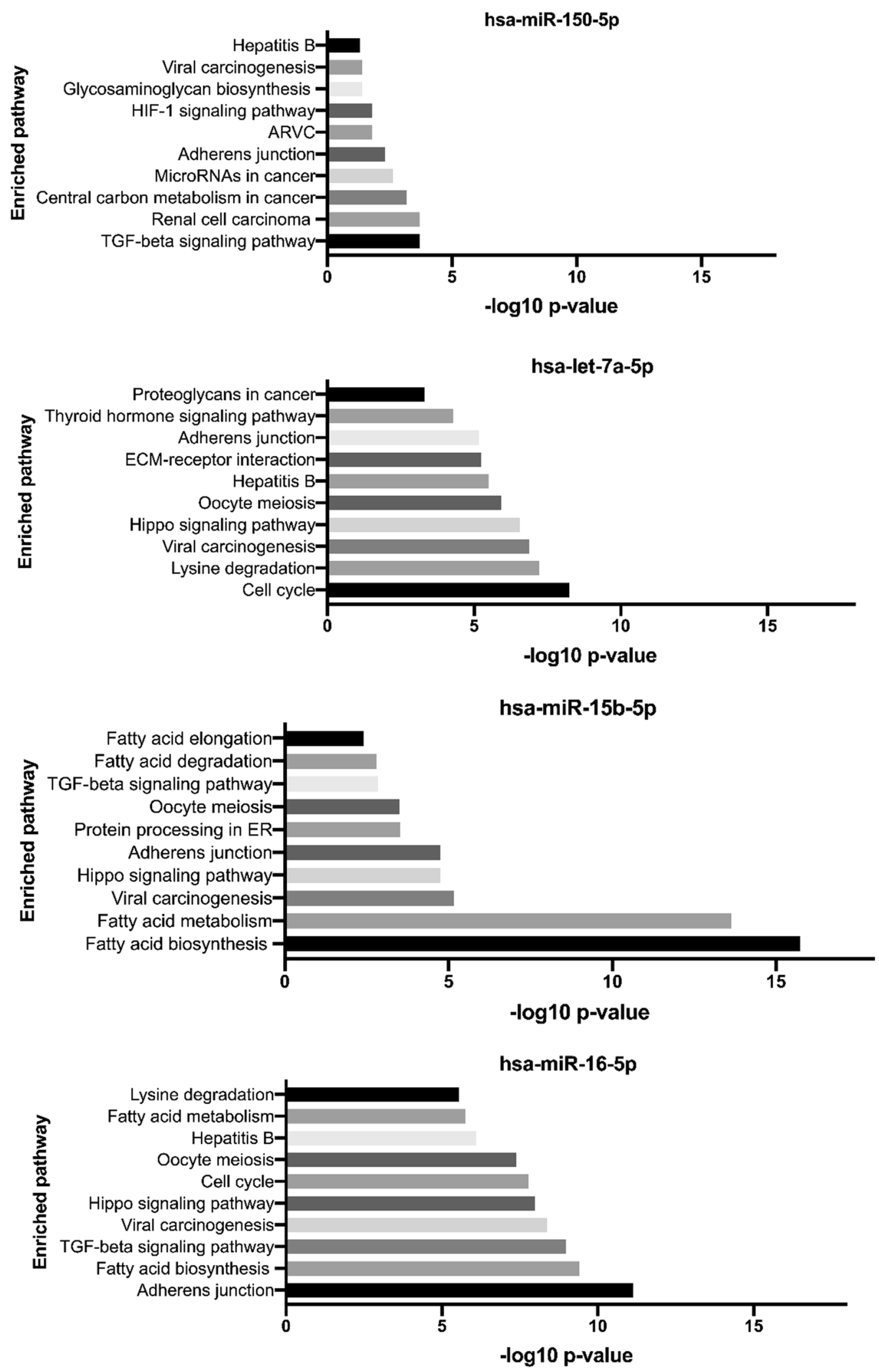

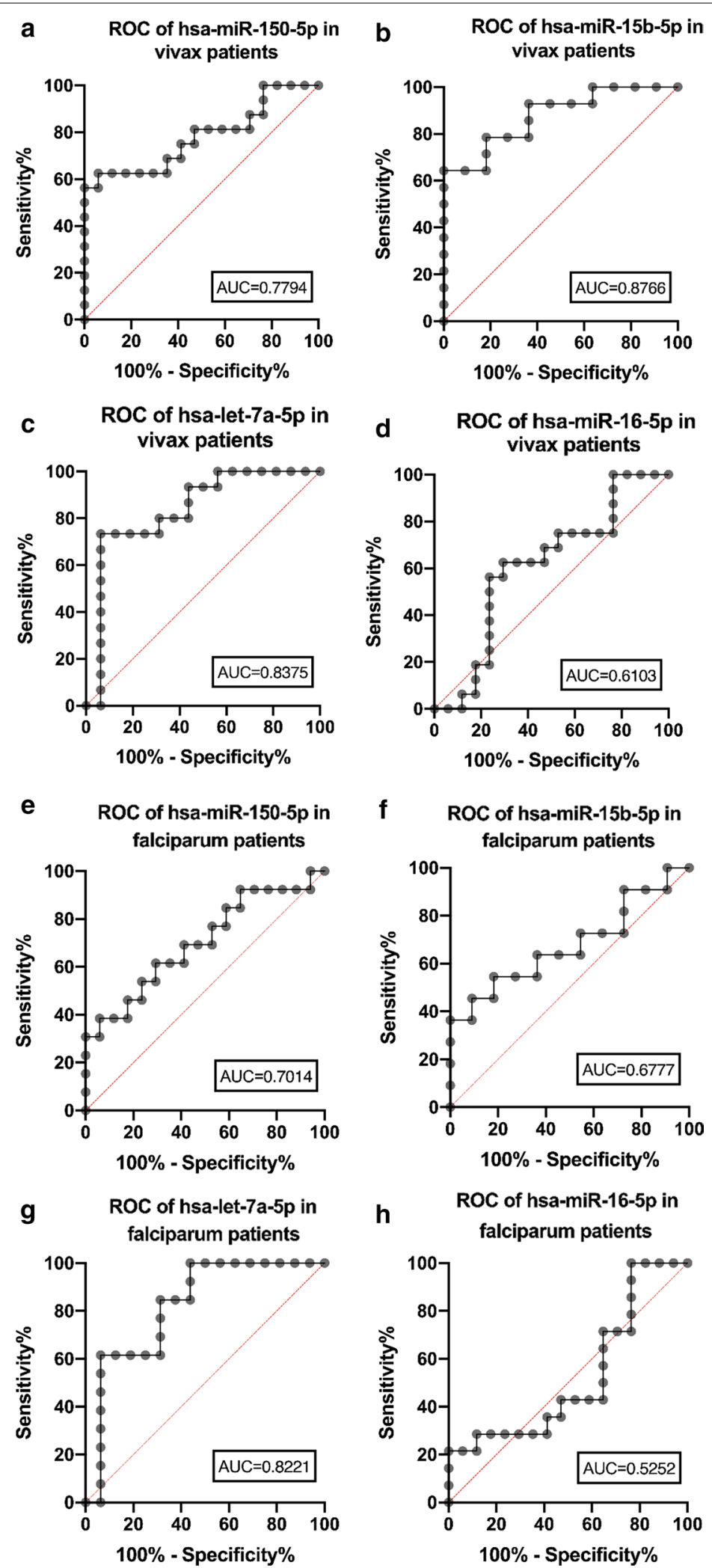

Fig. 5 Area Under the Receiver Operating Characteristic (ROC) curve (AUC) analysis. The AUC of each tests presents with the maximum AUC. In P. vivax-infected patients, hsa-miR-150-5p AUC was 0.7794 ( $p=0.0062)$, hsa-miR-15b-5p AUC was 0.8766 ( $p=0.0015)$, and hsa-let-7a was 0.8375 $(p=0.0014)$. For the P. falciparum-infected patients, there was the hsa-let-7a-5p that was statistically significant with AUC $0.8221(p=0.0033)$ 
with Ago2 [41] could be detected in P. falciparum and potentially targets the Plasmodium gene: Rad54 [37]. In addition, hsa-let-7a expression was low in erythrocyte-derived EVs in a $P$. falciparum in vitro experiment but another member in the let7 family: hsa-let-7b was higher in the EVs fraction compared to infected or uninfected erythrocytes. Interestingly, miRNAs profiling from in vitro $P$. falciparum infected erythrocyte-derived EVs also showed that hsa-let-7a, hsa-let-7i, hsa-let-7 g and hsa-let-7f were highly expressed [42]. Some of these miRNAs might derive from parasitized red blood cells as they were found highly enriched in previous analyses [33]. In addition, a study in experimental cerebral malaria demonstrated an increased expression of let-7i in brain tissues which might link to cerebral malaria pathogenesis [39]. This implies that miRNAs within the let-7 family might play a role in the parasite biology, malaria pathogenesis and further studies should be performed to elucidate their functions.

Furthermore, the relative expression of hsa-miR-16-5p was analysed. There was no change in the abundance both in $P$. vivax-infected patients and $P$. falciparuminfected patients when compared to uninfected individuals. This is consistent with two previous studies showing that hsa-miR-16-5p is highly expressed in both $P$. falciparum-infected erythrocytes and normal erythrocytes. Thus, the relative expression of this miRNA might not be modulated during infection. However, these results differ from a study that found down-regulation of hsa-miR16-5p in $P$. vivax-infected patients [44]. Interestingly, a previous study using an ECM model (P. berghei strain ANKA) found an up-regulation of miR-16 in plasmaderived microvesicles [47] suggesting that further studies are needed to elucidate the expression of this miRNA during complicated and uncomplicated malaria infection.

Several pathways might be important in the context of malaria. Importantly, the adherens junction and the transforming growth factor (TGF)- $\beta$ were found enriched by the targeted genes of 3 dysregulated miRNAs. Adherens junctions are possibly regulated by hsa-miR-150-5p, hsa-miR-15b-5p and hsa-let-7a-5p. The blood-brain barrier (BBB) is a vital compartment of central nervous system as it separates the CNS from surrounding environment. Adherens junctions in endothelial cells participate to the forming and maintaining of the integrity of the BBB. A number of studies also showed that some miRNAs might regulate this type of junction. For example, the down regulation of vascular endothelium cadherin (VE-Cadherin) was affected by overexpression of miR-101 and this lead to HIV-associated neurological disorder [57]. Also, the overexpression of miR-142-3p repressed the expression of VE-Cadherin and impaired vascular integrity in zebrafish [58]. Similarly, in ECM, the authors postulated the roles of overexpressed miR-19a-3p and miR-19b-5p in this pathway as well [40]. Knowing the roles of miRNAs in the context of malaria particularly cerebral malaria pathogenesis is paramount as it might lead to the development of an adjunctive therapy. For instance, inhibition of miR-27 could prevent vascular leakage associated with ischaemia [59]. However, no study of the dysregulated miRNAs analysed in this work, which are in association with this pathway, has been performed in human malaria. More studies are therefore needed to fill this gap.

Circulating miRNAs have been studied and proposed as diagnostic biomarkers in many infectious diseases including malaria. Most studies on infectious diseases have detected human miRNAs such as those in tuberculosis [60], hepatitis B [61], schistosomiasis [62] while some studies investigated microbial miRNAs as biomarkers as well [54, 63-65]. Despite the fact that Plasmodium spp. lack RNA interference machinery and its own miRNAs [32, 36], human derived miRNAs were demonstrated as promising biomarkers [44, 48]. For example, in human malaria, hsa-miR-16 and hsa-miR-451a were proposed to be biomarkers for $P$. vivax infection diagnosis [44]. In the present study, the potential of miRNAs isolated from EVs in malaria patients was evaluated for the first time. The calculated p-values from AUC analysis indicated that hsa-miR-150-5p, hsa-miR-15b-5p might be used as biomarkers for $P$. vivax malaria whereas hsalet-7a-5p might be used to test for both $P$. vivax and $P$. falciparum malaria. However, the sensitivity and specificity were not much higher. Therefore, further analysis of these miRNAs is recommended because the number of patient samples were relatively low in this study. Furthermore, as mentioned earlier, this study selected the miRNAs based on previous studies that investigated these miRNAs in the context of malaria. A more in-depth study is needed to develop new biomarkers. For instance, profiling miRNA using microarrays or next-generation sequencing will allow an evaluation of all miRNAs present in the EVs. Also, these miRNAs should be analysed in the patients after they recover from the disease. It might be useful in the context of $P$. vivax malaria as this species can cause relapse infection. In addition, these markers should be further analysed and compared in the different groups of $P$. falciparum malaria patients such as uncomplicated and severe malaria. These will be useful if patients can be early predicted the chance of developing severe malaria beforehand.

\section{Conclusion}

This novel study explored hsa-miR-150-5p, hsa-miR15b-5p, hsa-let-7a-5p, and hsa-miR-16-5p which were isolated from EVs from Thai malaria patient's plasma. 
The relative expression of hsa-miR-150-5p and hsa-miR15b-5p were significantly higher in $P$. vivax-infected patients where hsa-let-7a-5p was significantly up-regulated in both $P$. vivax-infected patients and $P$. falciparum-infected patients. Targets prediction and pathways enrichment analysis also provided the possible roles of these up-regulated miRNAs in the context of malaria, especially the TGF- $\beta$ pathway, which need further investigation to elucidate their exact roles in the malaria biology and the disease pathogenesis.

As this current study only evaluated those miRNAs from EVs of uncomplicated malaria patients, it is therefore encouraging to analyse in the future the EVs-derived miRNAs in those patients with severe complications.

\section{Supplementary information}

Supplementary information accompanies this paper at https://doi. org/10.1186/s12936-020-03360-z.

Additional file 1: Table S1. Individual target prediction of up-regulated miRNAs and genes involved in malaria pathway.

\section{Acknowledgements}

The authors acknowledge Assistant Professor Tewarit Sarachana and Miss Surangrat Thongkorn for their invaluable advice in the data analysis.

\section{Authors' contributions}

NK, ISC, MI, VC and DP designed and conceived the study. ISC, FD and NT designed the qPCR assays for the paper. ISC and FD performed the experimental studies. NK, ISC and VC contributed to the data analysis. NK performed bioinformatic analysis, interpreted and wrote the manuscript. All authors provided advice in figure preparation and manuscript revision. All authors read and approved the final manuscript.

\section{Funding}

This research was funded by Chulalongkorn University and Thailand Science Research Innovation (TSRI), RTA6280006. Nutpakal Ketprasit was financially supported both tuition fees and monthly stipend by the scholarship from Graduate School, Chulalongkorn University to commemorate the 72nd Anniversary of his Majesty King Bhumibol Adulyadej of 2016 academic year. For research expense, Nutpakal was funded by the 90th Anniversary Chulalongkorn University Fund (Ratchadaphiseksomphot Endowment Fund: GCUGR1125602095M) of 2016 academic year and Overseas research scholarship of 2016 academic year supported by Graduate school and Faculty of Allied Health Sciences, Chulalongkorn University. Iris Simone Cheng is supported by the Australian Government Research Training Program Stipend.

\section{Availability of data and materials}

The datasets used and/or analysed during the current study are available from the corresponding authors on reasonable request.

\section{Ethical approval}

Ethics approval by Ethics committee of the Faculty of Tropical Medicine, Mahidol University, MUTM 2012-046-05. Ethics approval for the University of Technology Sydney, UTS HREC ETH18-2756.

\section{Consent for publication}

All authors reviewed and consented the final manuscript for publication.

\section{Competing interests}

All authors declare no competing interests, no personal or professional conflicts of interest, and no financial support from the companies that produce and/or distribute the devices, or materials described in this report.

\section{Author details}

${ }^{1}$ Graduate Programme in Clinical Hematology Sciences, Department of Clinical Microscopy, Faculty of Allied Health Sciences, Chulalongkorn University, Bangkok, Thailand. ${ }^{2}$ Malaria and Microvesicles Research Group, School of Life Sciences, Faculty of Sciences, University Technology of Sydney, Ultimo, Sydney, NSW 2007, Australia. ${ }^{3}$ Non-coding RNA Cancer Group, School of Biomedical Engineering, Faculty of Engineering and IT, University Technology of Sydney, Sydney, NSW, Australia. ${ }^{4}$ Department of Molecular Tropical Medicine and Genetics, Faculty of Tropical Medicine, Mahidol University, Bangkok, Thailand. ${ }^{5}$ Oxidation in Red Cell Disorders Research Unit, Department of Clinical Microscopy, Faculty of Allied Health Sciences, Chulalongkorn University, 154 Rama 1 Road, Pathumwan, Bangkok 10330, Thailand.

Received: 21 March 2020 Accepted: 5 August 2020

Published online: 10 August 2020

\section{References}

1. World Health Organization. World malaria report 2019. Geneva: World Health Organization; 2019.

2. White NJ. Malaria. In: Manson's Tropical Diseases, 23rd Edn. Farrar J, Hotez P, Thomas Junghanss T, Kang G, Lalloo D, White NJ, Eds. Saunders Ltd Publ.; 2014.

3. Ashley EA, Dhorda M, Fairhurst RM, Amaratunga C, Lim P, Suon S, et al. Spread of artemisinin resistance in Plasmodium falciparum malaria. N Engl J Med. 2014;371:411-23.

4. Hamilton WL, Amato R, van der Pluijm RW, Jacob CG, Quang HH, ThuyNhien NT, et al. Evolution and expansion of multidrug-resistant malaria in southeast Asia: a genomic epidemiology study. Lancet Infect Dis. 2019;19:943-51.

5. Andaloussi SE, Mäger I, Breakefield XO, Wood MJ. Extracellular vesicles: biology and emerging therapeutic opportunities. Nat Rev Drug Discov. 2013;12:347-57.

6. Caruso S, Poon IKH. Apoptotic cell-derived extracellular vesicles: more than just debris. Front Immunol. 2018;9:1486.

7. György B, Szabó TG, Pásztói M, Pál Z, Misják P, Aradi B, et al. Membrane vesicles, current state-of-the-art: emerging role of extracellular vesicles. Cell Mol Life Sci. 2011;68:2667-88.

8. Hugel B, Martínez MC, Kunzelmann C, Freyssinet J-M. Membrane microparticles: two sides of the coin. Physiology. 2005;20:22-7.

9. Hugel B, Socié G, Vu T, Toti F, Gluckman E, Freyssinet J-M, et al. Elevated levels of circulating procoagulant microparticles in patients with paroxysmal nocturnal hemoglobinuria and aplastic anemia. Blood. 1999;93:3451-6.

10. Nantakomol D, Dondorp AM, Krudsood S, Udomsangpetch R, Pattanapanyasat K, Combes V, et al. Circulating red cell-derived microparticles in human malaria. J Infect Dis. 2011;203:700-6.

11. Combes V, Taylor TE, Juhan-Vague I, Mege JL, Mwenechanya J, Tembo $M$, et al. Circulating endothelial microparticles in malawian children with severe falciparum malaria complicated with coma. JAMA. 2004;291:2542-4.

12. Iraci N, Leonardi T, Gessler F, Vega B, Pluchino S. Focus on extracellular vesicles: physiological role and signalling properties of extracellular membrane vesicles. Int J Mol Sci. 2016;17:171.

13. Schorey JS, Cheng Y, Singh PP, Smith VL. Exosomes and other extracellular vesicles in host-pathogen interactions. EMBO Rep. 2015;16:24-43.

14. Shah R, Patel T, Freedman JE. Circulating extracellular vesicles in human disease. N Engl J Med. 2018;379:958-66.

15. Mfonkeu JBP, Gouado I, Kuate HF, Zambou O, Zollo PHA, Grau GER, et al. Elevated cell-specific microparticles are a biological marker for cerebral dysfunctions in human severe malaria. PLoS ONE. 2010;5:e13415.

16. El-Assaad F, Wheway J, Hunt NH, Grau GE, Combes V. Production, fate and pathogenicity of plasma microparticles in murine cerebral malaria. PLoS Pathog. 2014;10:e1003839.

17. Combes V, Coltel N, Alibert M, Van Eck M, Raymond C, Juhan-Vague I, et al. ABCA1 gene deletion protects against cerebral malaria: potential pathogenic role of microparticles in neuropathology. The American journal of pathology. 2005;166(1):295-302. 
18. Campos FMF, Franklin BS, Teixeira-Carvalho A, Filho ALS, de Paula SCO, Fontes CJ, et al. Augmented plasma microparticles during acute Plasmodium vivax infection. Malar J. 2010;9:327.

19. Martin-Jaular L, Nakayasu ES, Ferrer M, Almeida IC, del Portillo HA. Exosomes from Plasmodium yoelii-infected reticulocytes protect mice from lethal infections. PLOS ONE. 2011;6:e26588.

20. Sahu U, Sahoo PK, Kar SK, Mohapatra BN, Ranjit M. Association of TNF level with production of circulating cellular microparticles during clinical manifestation of human cerebral malaria. Hum Immunol. 2013;74:713-21.

21. Tiberti N, Latham SL, Bush S, Cohen A, Opoka RO, John CC, et al. Exploring experimental cerebral malaria pathogenesis through the characterisation of host-derived plasma microparticle protein content. Sci Rep. 2016;6:37871.

22. Couper KN, Barnes T, Hafalla JCR, Combes V, Ryffel B, Secher T, et al. Parasite-derived plasma microparticles contribute significantly to malaria infection-induced inflammation through potent macrophage stimulation. PLoS Pathog. 2010;6:e1000744.

23. Ye W, Chew M, Hou J, Lai F, Leopold SJ, Loo HL, et al. Microvesicles from malaria-infected red blood cells activate natural killer cells via MDA5 pathway. PLoS Pathog. 2018;14:e1007298.

24. Mantel P-Y, Hoang Anh N, Goldowitz I, Potashnikova D, Hamza B, Vorobjev I, et al. Malaria-infected erythrocyte-derived microvesicles mediate cellular communication within the parasite population and with the host immune system. Cell Host Microbe. 2013;13:521-34.

25. Regev-Rudzki N, Wilson DW, Carvalho TG, Sisquella X, Coleman BM, Rug $\mathrm{M}$, et al. Cell-cell communication between malaria-infected red blood cells via exosome-like vesicles. Cell. 2013;153:1120-33.

26. Correa R, Coronado L, Caballero Z, Faral P, Robello C, Spadafora C. Extracellular vesicles carrying lactate dehydrogenase induce suicide in increased population density of Plasmodium falciparum in vitro. Sci Rep. 2019;9:5042.

27. Sisquella X, Ofir-Birin Y, Pimentel MA, Cheng L, Abou Karam P, Sampaio $N G$, et al. Malaria parasite DNA-harbouring vesicles activate cytosolic immune sensors. Nat Commun. 2017:8:1985.

28. Cheng L, Sharples RA, Scicluna BJ, Hill AF. Exosomes provide a protective and enriched source of miRNA for biomarker profiling compared to intracellular and cell-free blood. J Extracell Vesicles. 2014. https://doi. org/10.3402/jev.v3.23743.

29. Vojtech L, Woo S, Hughes S, Levy C, Ballweber L, Sauteraud RP, et al. Exosomes in human semen carry a distinctive repertoire of small noncoding RNAs with potential regulatory functions. Nucleic Acids Res. 2014;42:7290-304

30. Valadi H, Ekström K, Bossios A, Sjöstrand M, Lee JJ, Lötvall JO. Exosomemediated transfer of mRNAs and microRNAs is a novel mechanism of genetic exchange between cells. Nat Cell Biol. 2007;9:654.

31. Lee RC, Feinbaum RL, Ambros V. The C. elegans heterochronic gene lin-4 encodes small RNAs with antisense complementarity to lin-14. Cell. 1993;75:843-54.

32. Rathjen T, Nicol C, McConkey G, Dalmay T. Analysis of short RNAs in the malaria parasite and its red blood cell host. FEBS Lett. 2006;580:5185-8.

33. Xue X, Zhang Q, Huang Y, Feng L, Pan W. No miRNA were found in Plasmodium and the ones identified in erythrocytes could not be correlated with infection. Malar J. 2008;7:47.

34. Coulson RMR, Hall N, Ouzounis CA. Comparative genomics of transcriptional control in the human malaria parasite Plasmodium falciparum. Genome Res. 2004;14:1548-54.

35. Hall N, Karras M, Raine JD, Carlton JM, Kooij TW, Berriman M, et al. A comprehensive survey of the Plasmodium life cycle by genomic, transcriptomic, and proteomic analyses. Science. 2005;307:82-6.

36. Baum J, Papenfuss AT, Mair GR, Janse CJ, Vlachou D, Waters AP, et al. Molecular genetics and comparative genomics reveal RNAi is not functional in malaria parasites. Nucleic Acids Res. 2009: gkp239.

37. Dandewad V, Vindu A, Joseph J, Seshadri V. Import of human miRNARISC complex into Plasmodium falciparum and regulation of the parasite gene expression. J Biosci. 2019:44:50.

38. LaMonte G, Philip N, Reardon J, Lacsina JR, Majoros W, Chapman L, et al. Translocation of sickle cell erythrocyte microRNAs into Plasmodium falciparum inhibits parasite translation and contributes to malaria resistance. Cell Host Microbe. 2012;12:187-99.
39. El-Assaad F, Hempel C, Combes V, Mitchell AJ, Ball HJ, Kurtzhals JA, et al. Differential microRNA expression in experimental cerebral and noncerebral malaria. Infect Immun. 2011;79:2379-84.

40. Martin-Alonso A, Cohen A, Quispe-Ricalde MA, Foronda P, Benito A, Berzosa $\mathrm{P}$, et al. Differentially expressed microRNAs in experimental cerebral malaria and their involvement in endocytosis, adherens junctions, FoxO and TGF- $\beta$ signalling pathways. Sci Rep. 2018;8:11277.

41. Mantel PY, Hjelmqvist D, Walch M, Kharoubi-Hess S, Nilsson S, Ravel D, et al. Infected erythrocyte-derived extracellular vesicles alter vascular function via regulatory Ago2-miRNA complexes in malaria. Nat Commun. 2016:7:12727

42. Babatunde KA, Mbagwu S, Hernandez-Castaneda MA, Adapa SR, Walch $M$, Filgueira $L$, et al. Malaria infected red blood cells release small regulatory RNAs through extracellular vesicles. Sci Rep. 2018;8:884.

43. Wang Z, Xi J, Hao X, Deng W, Liu J, Wei C, et al. Red blood cells release microparticles containing human argonaute 2 and miRNAs to target genes of Plasmodium falciparum. Emerg Microbes Infect. 2017;6:e75.

44. Chamnanchanunt S, Kuroki C, Desakorn V, Enomoto M, Thanachartwet V, Sahassananda D, et al. Downregulation of plasma miR-451 and miR-16 in Plasmodium vivax infection. Exp Parasitol. 2015;155:19-25.

45. Prapansilp Panote TG. MicroRNA in malaria. MicroRNAs in medicine. Lawrie CH, Ed. Wiley; 2013:183-97.

46. van Loon W, Gai PP, Hamann L, Bedu-Addo G, Mockenhaupt FP. MiRNA146a polymorphism increases the odds of malaria in pregnancy. Malar J. 2019;18:7.

47. Cohen A, Zinger A, Tiberti N, Grau GE, Combes V. Differential plasma microvesicle and brain profiles of microRNA in experimental cerebral malaria. Malar J. 2018;17:192.

48. Kaur H, Sehgal R, Kumar A, Sehgal A, Bansal D, Sultan AA. Screening and identification of potential novel biomarker for diagnosis of complicated Plasmodium vivax malaria. J Transl Med. 2018;16:272.

49. de Ronde MWJ, Ruijter JM, Moerland PD, Creemers EE, Pinto-Sietsma SJ. Study design and qPCR data analysis guidelines for reliable circulating miRNA biomarker experiments: a Review. Clin Chem. 2018;64:1308-18.

50. Livak KJ, Schmittgen TD. Analysis of relative gene expression data using real-time quantitative PCR and the 2(-Delta Delta C(T)) Method. Methods. 2001;25:402-8

51. Agarwal V, Bell GW, Nam J-W, Bartel DP. Predicting effective microRNA target sites in mammalian mRNAs. eLife. 2015;4:e05005.

52. Kanehisa M, Goto S. KEGG: Kyoto encyclopedia of genes and genomes. Nucleic Acids Res. 2000;28:27-30.

53. Vlachos IS, Zagganas K, Paraskevopoulou MD, Georgakilas G, Karagkouni D, Vergoulis T, et al. DIANA-miRPath v3.0: deciphering microRNA function with experimental support. Nucleic Acids Res. 2015;43:W460-6.

54. Meningher T, Lerman G, Regev-Rudzki N, Gold D, Ben-Dov IZ, Sidi Y, et al. Schistosomal microRNAs isolated from extracellular vesicles in sera of infected patients: a new tool for diagnosis and follow-up of human schistosomiasis. J Infect Dis. 2016;215:378-86.

55. Obaldia N, Meibalan E, Sa JM, Ma S, Clark MA, Mejia P, et al. Bone marrow is a major parasite reservoir in Plasmodium vivax infection. mBio. 2018;9:e00625-718.

56. Baro B, Deroost K, Raiol T, Brito M, Almeida AC, de Menezes-Neto A, et al. Plasmodium vivax gametocytes in the bone marrow of an acute malaria patient and changes in the erythroid miRNA profile. PLoS Negl Trop Dis. 2017;11:e0005365.

57. Mishra R, Singh SK. HIV-1 Tat C modulates expression of miRNA-101 to suppress VE-cadherin in human brain microvascular endothelial cells. J Neurosci. 2013;33:5992-6000

58. Lalwani MK, Sharma M, Singh AR, Chauhan RK, Patowary A, Singh N, et al. Reverse genetics screen in zebrafish identifies a role of miR-142a-3p in vascular development and integrity. PLoS ONE. 2012;7:e52588.

59. Young JA, Ting KK, Li J, Moller T, Dunn L, Lu Y, et al. Regulation of vascular leak and recovery from ischemic injury by general and VE-cadherinrestricted miRNA antagonists of miR-27. Blood. 2013;122:2911-9.

60. Qi Y, Cui L, Ge Y, Shi Z, Zhao K, Guo X, et al. Altered serum microRNAs as biomarkers for the early diagnosis of pulmonary tuberculosis infection. BMC Infect Dis. 2012;12:384.

61. Zhang X, Zhang Z, Dai F, Shi B, Chen L, Zhang X, et al. Comparison of circulating, hepatocyte specific messenger RNA and microRNA as biomarkers for chronic hepatitis B and C. PLOS ONE. 2014;9:e92112. 
62. He $X$, Sai $X$, Chen $C$, Zhang $Y, X u X$, Zhang D, et al. Host serum miR-223 is a potential new biomarker for Schistosoma japonicum infection and the response to chemotherapy. Parasit Vectors. 2013;6:272.

63. Alizadeh Z, Mahami-Oskouei M, Spotin A, Kazemi T, Ahmadpour E, Cai P, et al. Parasite-derived microRNAs in plasma as novel promising biomarkers for the early detection of hydatid cyst infection and post-surgery follow-up. Acta Trop. 2019;202:105255.

64. Quintana JF, Makepeace BL, Babayan SA, Ivens A, Pfarr KM, Blaxter M, et al. Extracellular Onchocerca-derived small RNAs in host nodules and blood. Parasit Vectors. 2015;8:58.
65. Tritten L, Burkman E, Moorhead A, Satti M, Geary J, Mackenzie C, et al. Detection of circulating parasite-derived MicroRNAs in filarial infections. PLoS Negl Trop Dis. 2014;8:e2971.

\section{Publisher's Note}

Springer Nature remains neutral with regard to jurisdictional claims in published maps and institutional affiliations.
Ready to submit your research? Choose BMC and benefit from:

- fast, convenient online submission

- thorough peer review by experienced researchers in your field

- rapid publication on acceptance

- support for research data, including large and complex data types

- gold Open Access which fosters wider collaboration and increased citations

- maximum visibility for your research: over 100M website views per year

At BMC, research is always in progress.

Learn more biomedcentral.com/submissions 